Music-Supported Training is More Efficient than Functional Motor Training for Recovery of Fine Motor Skills in Stroke Patients

Author(s): Sabine Schneider, Thomas Münte, Antoni Rodriguez-Fornells, Michael Sailer, Eckart Altenmüller

Reviewed work(s):

Source: Music Perception: An Interdisciplinary Journal, Vol. 27, No. 4 (April 2010), pp. 271280

Published by: University of California Press

Stable URL: http://www.jstor.org/stable/10.1525/mp.2010.27.4.271

Accessed: 03/01/2012 05:19

Your use of the JSTOR archive indicates your acceptance of the Terms \& Conditions of Use, available at http://www.jstor.org/page/info/about/policies/terms.jsp

JSTOR is a not-for-profit service that helps scholars, researchers, and students discover, use, and build upon a wide range of content in a trusted digital archive. We use information technology and tools to increase productivity and facilitate new forms of scholarship. For more information about JSTOR, please contact support@jstor.org. 


\section{Music-Supported Training is More Efficient Than Functional Motor Training for Recovery of Fine Motor Skills in Stroke Patients}

\author{
Sabine SCHNeIDER \\ University of Music and Drama, Hanover, Germany \\ Thomas MÜnte AND \\ Antoni RodrigueZ-Fornells \\ Otto von Guericke University, Magdeburg, German \\ Michael Sailer \\ MEDIAN Klinik NRZ, Magdeburg, Germany \\ Eckart Altenmüller \\ University of Music and Drama, Hanover, Germany
}

MOTOR IMPAIRMENTS ARE COMMON AFTER STROKE but efficacious therapies for these dysfunctions are scarce. Extending an earlier study on the effects of music-supported training (MST), behavioral indices of motor function were obtained before and after a series of training sessions to assess whether this new treatment leads to improved motor functions. Furthermore, music-supported training was contrasted to functional motor training according to the principles of constraint-induced therapy (CIT). In addition to conventional physiotherapy, 32 stroke patients with moderately impaired motor function and no previous musical experience received 15 sessions of MST over a period of three weeks, using a manualized, step-bystep approach. A control group consisting of 15 patients received 15 sessions of CIT in addition to conventional physiotherapy. A third group of 30 patients received exclusively conventional physiotherapy and served as a control group for the other three groups. Fine as well as gross motor skills were trained by using either a MIDI-piano or electronic drum pads programmed to emit piano tones. Motor functions were assessed by an extensive test battery. MST yielded significant improvement in fine as well as gross motor skills with respect to speed, precision, and smoothness of movements. These improvements were greater than after CIT or conventional physiotherapy. In conclusion, with equal treatment intensity, MST leads to more pronounced improvements of motor functions after stroke than CIT.
Received February 3, 2010, accepted February 23, 2010.

Key words: neurorehabilitation, music-supported training, stroke, functional motor training, auditorysensory-motor integration

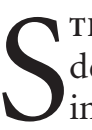
TROKE IS A LEADING CAUSE OF DISABILITY IN developed countries, affecting about 150,000 individuals per year in Germany. Nearly $90 \%$ of these patients suffer from motor impairments, which frequently prevent participation in everyday activities and limit vocational rehabilitation. Because the effectiveness of classic approaches, for example, those developed by Bobath (1948) has been found to be quite limited, there is an urgent need for more effective motor rehabilitation approaches (Langhammer \& Stanghelle, 2000; Woldag \& Hummelsheim, 2002). In this regard, data have accumulated indicating that repetitive massed practice of movements leads to improvements of motor control (Sterr et al., 2002). For example, in constraint-induced therapy (CIT), use of the impaired extremity is enforced by shaping procedures, often combined with immobilization of the healthy extremity for several hours per day. This procedure has been shown to lead to functional reorganization as demonstrated by transcranial magnetic stimulation and PET (Liepert, Bauder, Miltner, Taub, \& Weiller, 2000). Other studies have shown that plastic reorganization of neuronal networks plays an important role in recovery after brain injuries, ischemic lesions after stroke, or degeneration processes (Kujala, Alho \& Naatanen, 2000; Rossini \& Pauri, 2000). In this context it is important to keep in mind that according to animal studies cortical plasticity is increased by the behavioral relevance of the stimulation and its motivational value (Buonomano \& Merzenich, 1998).

Over the last several years we have developed a new approach to facilitate motor recovery using neurological music therapy, taking into account the above mentioned principles of repetitive training and increased motivational drive due to the inherent rewarding value

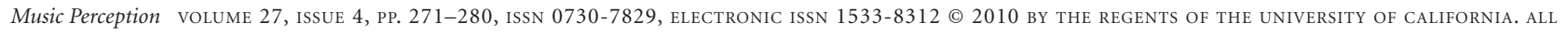
RIGHTS RESERVED. PLEASE DIRECT ALL REQUESTS FOR PERMISSION TO PHOTOCOPY OR REPRODUCE ARTICLE CONTENT THROUGH THE UNIVERSITY OF CALIFORNIA PRESS'S RIGHTS AND PERMISSIONS WEBSITE, HTTP://WWW.UCPRESSJOURNALS.COM/REPRINTINFO.ASP. DOI:10.1525/MP.2010.27.4.271 
of making music (Altenmüller, Schneider, MarcoPallares, \& Münte, 2009; Schneider, Schönle, Altenmüller, \& Münte, 2007). In addition to movement repetition and auditory feedback about the movement precision, the rapid establishment of auditory-sensorimotor corepresentations during the playing process has been hypothesized to underlie the efficacy of this treatment approach. Supporting evidence for such a mechanism comes from several studies, demonstrating that corresponding neuronal networks in auditory and motor brain areas integrate the processing of both modalities (Bangert \& Altenmüller, 2003; Bangert et al., 2006; Lahav, Saltzman, \& Schlaug, 2007). For example, in a longitudinal study, it was possible to follow the formation of such neuronal multisensory connections during piano training by beginner pianists. Nonmusicians were trained on a computer piano twice a week over a period of five weeks. They listened to short piano melodies $3 \mathrm{~s}$ in duration played in a five-tone range, and were then required, after a brief pause, to replay the melodies as accurately as possible with their right hand. After 20 mins of training, the first signs of increased neuronal coupling between auditory and motor brain regions were observable. After five weeks, listening to piano tunes produced additional activity in the central and left sensorimotor regions. Playing on a mute (soundless) keyboard produced additional activity in the auditory regions of both temporal lobes (Bangert \& Altenmüller, 2003). This experiment impressively demonstrated how dynamic brain adaptations accompany sensorimotor learning.

In light of the above mentioned studies on auditorysensorimotor coupling, we designed a training program that entailed active music making in the rehabilitation of stroke patients (Altenmüller et al., 2009; Schneider et al., 2007) according to the following principles:

- Repetition: Repetitive exercising of simple finger and arm movements.

- Auditory Feedback: Reinforcement of movement effects due to immediate auditory feedback supporting the precise timing control of movements.

- Shaping: Adapting the complexity of the required movements according to the individual progresses.

- Emotion: Increased motivation of the patients due to the playfulness and emotional impact of making music and acquiring a new skill.

In the present article we want to present our therapy program and summarize our data on its effectiveness. The current publication is an extension of recent data
(Altenmüller et al., 2009) showing a comparison of a second control group receiving Functional Motor Training according to CIT principles (Taub et al., 1993).

\section{Method}

In addition to conventional physiotherapy in a rehabilitation hospital, patients in the intervention group received daily sessions in which they produced tones, scales, and simple melodies on an electronic piano or an electronic drum set (emitting piano tones). As a control, a smaller group of patients received, in addition to conventional physiotherapy, functional motor training according to CIT principles (Taub et al., 1993).

\section{Patients}

This study was primarily designed as a two group parallel study. Later, a third group was added to compare the effects between the first two groups to an established neurorehabilitation technique, and to control for the time therapists spent with patients.

Seventy-seven inpatients from a neurological rehabilitation hospital participated in the study. All suffered from moderate impairment of motor function of upper extremities following stroke.

Inclusion criteria were specified similar to those adopted for CIT (Taub et al., 1993). In particular, (a) patients had to have residual function of the affected extremity; that is, the ability to move the affected arm without help from the healthy side, and to move the index finger without help from the healthy hand. Moreover, (b) patients had to score over 50 overall on the Barthel Index (possible score 100; Mahoney \& Barthel, 1965), and (c) performance on the Nine Hole Pegboard Test had to be slower than that of the mean minus $2 S D$ of the healthy control group (mean peg/s = $0.68, S D=0.14$; Heller et al., 1987). Patients were assigned randomly (by occupational therapists not involved in the study) to two groups receiving either conventional treatment only ( $n=30$; henceforth CG), or music-supported therapy in addition to conventional therapy $(n=32$; henceforth MG). Later, following Taub et al. (1993), the CIT group was recruited in the order of admission to the rehabilitation clinic without specific additional selection criteria ( $n=15$; henceforth TG) in order to exclude bias in patient recruitment.

Table 1 shows pertinent clinical and demographic data. Except for the gender distribution and extremity affected, no significant differences were found between MG, CG, and TG. The majority of left-hemisphere 
TABLE 1. Clinical and Demographic Data for Groups MG, CG, and TG.

\begin{tabular}{lccc}
\hline Group & MG & CG & TG \\
& $(n=32)$ & $(n=30)$ & $(n=15)$ \\
\hline Gender (male/female) & $16 / 16$ & $24 / 6$ & $10 / 5$ \\
Age $(M \pm S D)$; Range (in years) & $55.7 \pm 12.3$ & $53 \pm 11.8$ & $56,1 \pm 10.7$ \\
& $(17-74)$ & $(30-77)$ & $(36-76)$ \\
Affected arm (left/right) & $17 / 15$ & $15 / 15$ & $4 / 11$ \\
Education in years $(M \pm S D)$ & $9.8 \pm 1.6$ & $9.1 \pm 1.3$ & $9.3 \pm 1.2$ \\
Handedness (right/left/ambidextrous) & $30 / 1 / 1$ & $28 / 1 / 1$ & $12 / 3$ \\
Months after onset & 1.9 & 1.9 & 1.7 \\
Barthel-Index $(M \pm S D)$ & $85.9 \pm 15.9$ & $84.3 \pm 16.0$ & $88.33 \pm 14.5$ \\
Ischemic stroke/Bleeding & $26 / 6$ & $27 / 3$ & $12 / 3$ \\
\hline
\end{tabular}

patients ( $n=8$ in MG, $n=9$ in CG, $\mathrm{n}=11$ in TG) showed an aphasia of differing syndromatology (Broca and Wernicke) and severity (mild to moderate).

In all three groups, motor abilities prior to therapies were comparable. Table 2 summarizes the motor measures taken as a baseline before therapy. No statistically significant differences were observed.

All patients were able to understand the instructions during assessments and therapy, and had been tested previously with the Aachener Aphasie Test (AAT) to determine speech capabilities. Eight additional potential participants were excluded because severe perceptual or cognitive deficits revealed by neuropsychological testing did not allow their participation. None of the remaining patients were diagnosed with depression or other psychiatric or neurological diseases. They were all native speakers of German. All 77 patients enrolled in the study completed the whole therapy and assessment program except for 8 patients (1 MG and 7 CG) who missed computerized movement analysis during post-treatment assessment.

Informed consent was obtained from each patient following detailed explanation of the study. The study was approved by the ethics review board of the University Hospital of Magdeburg.

\section{Procedures}

EVALUATION OF MOTOR FUNCTIONS

Motor functions were assessed prior to and after the treatment for CG, TG, and MG using the following four established measurement instruments, all of which are widely used in motor rehabilitation of the upper extremity:

TABLE 2. Mean Scores (SD) of the Pre-Testing of Motor Functions Between Groups.

\begin{tabular}{|c|c|c|c|c|}
\hline Motor test/Parameter & MG & CG & TG & $F(2,74)$ \\
\hline Box \& Block Test (score) & $25.1(17.6)$ & $30.8(21.0)$ & $30.5(16.2)$ & .44 \\
\hline Nine Hole Pegboard Test (score) & $4.1(4.0)$ & $4.9(4.1)$ & $4.9(3.8)$ & .72 \\
\hline Action Research Arm Test (score) & $33.3(23.9)$ & $36.4(23.3)$ & $42.7(19.6)$ & .43 \\
\hline Armparese Score Wade (score) & $4.5(2.8)$ & $4.7(2.8)$ & $5.2(2.5)$ & .74 \\
\hline Motor test/Parameter & MG & CG & TG & $F(2,66)$ \\
\hline Finger tapping FREQ in $\mathrm{Hz}$ & $2.0(1.4)$ & $1.6(1.4)$ & $2.0(1.4)$ & .94 \\
\hline Finger tapping VMAX in $\%$ & $160.2(105.5)$ & $126.8(112.1)$ & $143.4(124.0)$ & .67 \\
\hline Finger tapping NIV ${ }^{\star}$ & $1.7(2.9)$ & $2.1(2.7)$ & $1.7(2.9)$ & .67 \\
\hline Hand tapping FREQ in $\mathrm{Hz}$ & $1.8(1.4)$ & $1.5(1.4)$ & $1.8(1.2)$ & .94 \\
\hline Hand tapping VMAX in $\%$ & $102.5(80.0)$ & $100.6(110.8)$ & $75.2(49.8)$ & .40 \\
\hline Hand tapping NIV ${ }^{\star}$ & $1.9(3.1)$ & $2.5(2.9)$ & $2.1(3.3)$ & .28 \\
\hline Diadochokinesis FREQ in $\mathrm{Hz}$ & $1.2(0.8)$ & $1.2(1.1)$ & $1.2(0.7)$ & .77 \\
\hline Diadochokinesis VMAX in $\% s$ & $398.8(354.7)$ & $423.1(462.2)$ & $345.7(272.2)$ & .47 \\
\hline Diadochokinesis NIV* & $2.0(3.2)$ & $2.3(2.6)$ & $2.0(2.8)$ & .99 \\
\hline Aiming $5 \mathrm{~cm} \mathrm{~V} 2$ in $\mathrm{mm} / \mathrm{s}$ & $456.7(232.1)$ & $479.4(373.9)$ & $497.6(263.9)$ & .74 \\
\hline Aiming $0.8 . \mathrm{cm} \mathrm{V} 2$ in $\mathrm{mm} / \mathrm{s}$ & $487.5(243.4)$ & $464.4(366.8)$ & $519.0(275.5)$ & .96 \\
\hline
\end{tabular}


(1) Box and Block Test BBT (Mathiowetz, Volland, Kashman, \& Weber, 1985). This test, consisting of a box with two compartments and 150 cubes, measures manual dexterity. The subject grasped one cube at a time and moved it from one compartment to the other. The number of cubes transported within one minute was scored for the paretic and healthy extremity.

(2) Nine Hole Pegboard Test-9HPT (Parker, Wade, \& Langton-Hewer, 1986). Patients picked up nine rods (32 $\mathrm{mm}$ long, $9 \mathrm{~mm}$ diameter) and placed them into holes of $10 \mathrm{~mm}$ diameter as fast as possible. The time needed for placing all nine rods was transformed to a point-score used in subsequent statistical analysis (10 points for 5 to $15 \mathrm{~s}, 9$ points for 15 and $25 \mathrm{~s}$, etc.). Zero points were given if the task could not be performed. We developed this scoring system based on observations with pilot patients to accommodate patients who needed an exceedingly long time for the task (or even failed to perform) during the pre-test.

(3) Action Research Arm Test-ARAT (Lyle, 1981). This test assessed pertinent functions of the upper extremities within four subtests: grasp, grip, pinch, and gross movement, each containing items arranged in hierarchical order or difficulty. The maximum possible score is 57.

(4) Arm Paresis Score (Wade, Langton-Hewer, Wood, Skilbeck, \& Ismail, 1983). This arm function test consists of seven simple tasks for the affected hand alone and both hands together (opening a jar of jam, drawing a line, picking up and releasing a $2 "$ and $1 / 2 "$ cylinder, drinking water from a glass, combing ones hair, and opening/closing a clothes pin). For each successful task, one point was awarded.

Additionally, we used computerized movement analysis as an objective measurement of movement quality. A computerized movement analysis system (CMS 50, Zebris, Isny, Germany) was used based on the continuous recording of ultrasound pulses from senders applied to the dorsal phalanges of the index finger between the distal and proximal interdigital joints (DIP and PIP), over the metacarpophalangeal (MCP) joint, and over the dorsum of the wrist joint. Sampling rate was set at $200 \mathrm{~Hz}$, resulting in a temporal resolution of $66 \mathrm{~Hz}$ per sender. Continuous calculation of the $3 \mathrm{D}$ positions of the three senders was done with commercially obtainable software (WinData 2.19.3x, Fa. Zebris). For details of movement registration and position of senders, see Hermsdörfer, Marquardt, Wack, and Mai (1999).

In brief, two self-paced tapping movements of the upper limb were tested on each hand: whole hand tapping and index finger tapping. Patients were instructed to move as fast as possible without an external pace-maker.
Additionally, a rapidly alternating movement comprising pronation and supination of the forearm was required (diadochokinesia). The importance of movement speed was stressed when giving the instructions. Data analysis (using software 3DA-Version 1.5(c) by C. Marquardt, Munich, Germany) was performed on five movement cycles, yielding the following measures for each task:

(a) Frequency (FREQ): number of full cycles/s.

(b) Number of inversions of velocity profiles (NIV) per movement segment. This is an established measure of the "smoothness" of the movements. Inversions with amplitudes less than $3 \%$ of maximal velocity were excluded. The best value that could be reached was 1 .

(c) Average maximum angular velocity (VMAX) in $\%$.

As suggested by Hermsdörfer et al. (1999) we omitted the first and the last movement cycles to exclude artefacts due to movement onset/offset or fatigue. The five selected movement cycles were determined by marking the movement onset of the second and the offset of the sixth cycle. Three such measurements were conducted per task. In a second step a segment analysis was made for the selected movement cycles. Data were averaged over the five cycles and three repetitions to yield individual subject measurements that were entered into the statistical analysis.

CONVENTIONAL THERAPY

All participants (CG, MG, and TG) received standard therapies according to the instructions of the attending neurologists, including individual physical therapy and individual occupational therapy using different materials and group therapies, each 30 mins in duration. MG patients received a mean 27.4 units, TG 28.0 units, and CG patients 27.2 of conventional therapies within the three week study period (duration of each unit 30 mins).

CIT ACCORDING TO TAUB

As a control for the MG intervention group, 15 patients received fifteen 30-minute sessions of functional motor training (CIT) according to Taub et al. (1993), in addition to the conventional physiotherapy during three weeks. Here, fine motor abilities of the hands were trained using functional movements such as grasping and manipulating various objects, jolleying screws, handling clothes pegs, and so on. We tried to keep motivational factors, such as the interest in participating in the motor tasks, constant in TG and MG. The degree of difficulty of the manipulations increased during the training procedure. The major difference of the functional motor training as compared to the music-supported training therefore was the lack of auditory feedback. Also note that the time spent on the intervention was 
equated with that used for music-supported therapy and thus was considerably shorter than usually used in clinical studies on the Taub method. The duration of the therapy used in the current study was chosen to yield comparable conditions for both intervention groups. Also, a therapy of 30 min duration is better compatible with clinical necessities in rehabilitation hospitals.

\section{MUSIC-SUPPORTED TRAINING}

For three weeks, MG participants received fifteen 30-minute sessions, administered individually, in addition to conventional physiotherapy. Two different input devices were used, a MIDI-piano and an electronic drum set consisting of eight pads, each $20 \mathrm{~cm}$ in diameter, arranged in front of the patient. The drum pads (designated by numbers 1-8) were used to produce piano ( $\left.G, A, B, C, D, E, F, G^{\prime}\right)$ rather than drum sounds. Similarly, the MIDI-piano was arranged in such a way that only eight white keys (G, A, B, C, D, E, F, G') could be played by the subject. This offers the advantage of an input device testing fine motor skills (piano) and another input instrument testing gross motor skills (drum set), while keeping the output constant. The different modules and the rules for progression from one level of difficulty to the next are described below. The training was applied and monitored by the first author. Each session was documented and recorded for later analysis.

From experience gathered in a number of pilot patients, a modular training regime with stepwise increase of complexity was designed. For drum training, patients were seated on a chair without armrests or in their own wheelchair in front of the eight drum pads. The height and proximity of the drum pads were individually adjustable, because at the beginning of the experiment only some of the participants were able to hit the drums with their extended arm, and some could only reach the lower drum pads (1-3-6-8). Each exercise first was played by the instructor (S.S.) and then was repeated by the patient. The instructor stood behind the patient and supported the affected extremity if necessary.

Similarly, patients were seated in front of the MIDI piano with the instructor sitting next to them or standing behind them (on the affected side). Again, an exercise first was demonstrated by the instructor and then repeated by the patient. Patients started with the affected upper extremity and then played with the affected and healthy hand together.

The training was adaptable to the needs of the patients in terms of the number of tones they were required to play, velocity, order, and limb used for playing. Furthermore, the degree of difficulty was systematically increased using 10 set levels. Every patient started the exercises (between 8 and 12 per session) at the lowest level by playing (hitting) single tones or the same tone on the same drum pad or key. If patients successfully managed this task, they continued on the next level; if not, the previous task was repeated.

In the subsequent levels, patients were required to use an increasing number of drum pads (or piano keys) until all eight tones could be played in varied sequences. The most difficult levels required patients to play the beginnings of children's or folk songs and finally songs consisting of five to eight tones with the paretic hand. Twenty different songs were available for the eight tones (e.g., Ode to Joy). Frequent repetitions of identical movements, which have been proven essential for motor learning, were required.

The measures derived from the motor test battery (computerized movement analysis, Action Research Arm Test, Arm Paresis Score, Box and Block Test, Nine Hole Pegboard Test) were used to assess the effect of the music-supported training and constituted the dependent variables. These were entered into an ANOVA design with group as between-subjects factors and time-point (pre vs. post) as the within-subjects factor. Group $\mathrm{x}$ time-point interaction effects were taken as evidence of differential effects of therapy in the groups. Moreover, to determine the size of the treatment effects Cohen's $d$ (Cohen, 1988) was computed for each group separately.

\section{Results}

Prior to treatment, none of the above mentioned behavioral parameters characterizing motor skills differed in the three groups (Table 2). After the three week intervention, there were significant improvements in groups TG and MG. Conventional physiotherapy in CG did not produce an improvement in most of the parameters assessed.

The detailed results for the Box and Block Test, the Nine-hole pegboard test, the Action Research Arm Test, the Arm-paresis score after Wade, and the computerized movement analysis are displayed in Table 3.

In Figure 1, the results for the Box and Block Test are displayed. In contrast to CG and TG, MG increased the number of cubes grasped by around 10 per min. Differences between MG, CG, and TG were highly significant, $F(2,74)=57.08, p<.001$.

The data comparing the increase in finger tapping frequency is visualized in Figure 2. MG but not TG and CG showed a clear improvement for finger tapping frequency from the first to the second assessment (FREQ). As can be seen in Table 3, improvements in 
TABLE 3. Mean Scores (SD) of the Clinical Motor Tests Before and After Training.

\begin{tabular}{|c|c|c|c|c|c|c|}
\hline \multirow[b]{2}{*}{ Motor tests } & \multicolumn{2}{|c|}{ MG } & \multicolumn{2}{|c|}{ CG } & \multicolumn{2}{|c|}{ TG } \\
\hline & Pre & Post & Pre & Post & Pre & Post \\
\hline Box \& Block Test** & $25.1(17.6)$ & $35.1(18.3)$ & $30.8(21.0)$ & $32.5(20.9)$ & $30.5(16.2)$ & $33.7(16.3)$ \\
\hline Nine Hole Pegboard Test* & $4.1(4.0)$ & $5.4(3.5)$ & $4.9(4.1)$ & $4.9(4.1)$ & $4.9(3.8)$ & $5.1(3.8)$ \\
\hline Action Research Arm Test ${ }^{* *}$ & $33.3(23.9)$ & $41.4(17.6)$ & $36.4(23.3)$ & $36.9(23.3)$ & $42.7(19.6)$ & $45.4(18.6)$ \\
\hline Armparese Score Wade ${ }^{\star}$ & $4.5(2.8)$ & $5.9(1.8)$ & $4.7(2.8)$ & $4.8(2.8)$ & $5.2(2.5)$ & $5.5(2.4)$ \\
\hline Finger tapping FREQ ${ }^{\star \star}$ & $2.0(1.4)$ & $2.8(1.5)$ & $1.6(1.4)$ & $1.6(1.5)$ & $2.0(1.4)$ & $1.9(1.2)$ \\
\hline Finger tapping $\mathrm{VMAX}^{*}$ & $160.2(105.5)$ & $216.9(105.1)$ & $126.8(112.1)$ & $112.7(114.0)$ & $143.4(124.0)$ & $138.7(98.5)$ \\
\hline Finger tapping $\mathrm{NIV}^{\star}$ & $1.7(2.9)$ & $1.3(3.6)$ & $2.1(2.7)$ & $2.4(2.7)$ & $1.7(2.9)$ & $1.8(2.9)$ \\
\hline Hand tapping FREQ ${ }^{*}$ & $1.8(1.4)$ & $2.4(1.6)$ & $1.5(1.4)$ & $1.4(1.6)$ & $1.8(1.2)$ & $1.9(1.3)$ \\
\hline Hand tapping $\mathrm{VMAX}^{\star}$ & $102.5(80.1)$ & $158.9(152.8)$ & $100.6(110.8)$ & $67.0(72.1)$ & $75.2(49.8)$ & $65.6(42.0)$ \\
\hline Hand tapping NIV $^{*}$ & $1.9(3.1)$ & $1.5(3.4)$ & $2.5(2.9)$ & $2.9(2.8)$ & $2.1(3.3)$ & $2.0(3.2)$ \\
\hline Pro-/Supination FREQ & $1.2(0.8)$ & $1.6(1.0)$ & $1.2(1.1)$ & $1.2(1.1)$ & $1.2(0.7)$ & $1.4(0.9)$ \\
\hline Pro-/Supination VMAX & $398.8(354.7)$ & $420.8(298.7)$ & $423.1(462.2)$ & $395.5(380.5)$ & $345.7(272.2)$ & $482.6(426.7)$ \\
\hline Pro-/Supination NIV* & $2.0(3.2)$ & $1.5(3.0)$ & $2.3(2.6)$ & $2.3(2.6)$ & $2.0(2.8)$ & $2.0(2.7)$ \\
\hline V2 Target Movement $5 \mathrm{~cm}^{\star *}$ & $456.7(232.1)$ & $614.1(276.8)$ & $479.4(373.9)$ & $463.1(373.6)$ & $497.6(263.9)$ & $541.8(278.0)$ \\
\hline V2 Target Movement $0.8 \mathrm{~cm}^{*}$ & $487.5(243.4)$ & $595.9(284.0)$ & $464.4(366.8)$ & $451.3(363.3)$ & $519.0(275.5)$ & $573.1(326.2)$ \\
\hline
\end{tabular}

Group by time-point interaction ${ }^{* *} p<.001,{ }^{*} p<.05$

MG patients are not only more pronounced compared to CG, but also compared to TG in most parameters. The MG patients showed a substantial improvement over time compared to the CG and TG patients in the Box and Block test, Nine Hole Pegboard test, Action Research Arm Test, and Arm Paresis Score. Differences between MG, CG, and TG were highly significant, $F(2,66)=6.66$, $p=.002$.

The advantage of MG over TG with respect to movement smoothness and movement frequency was mostly restricted to finger movements. With respect to movements of the wrist or the forearm, as assessed by the hand tapping and the diadochokinesis test, CG and TG did not differ substantially in effect, and effects were generally less prominent, as can be seen in the calculation of effect sizes (Table 4).

Effect sizes were determined using Cohen's $d$. In the literature, effect sizes of 0.2 are considered small, effect

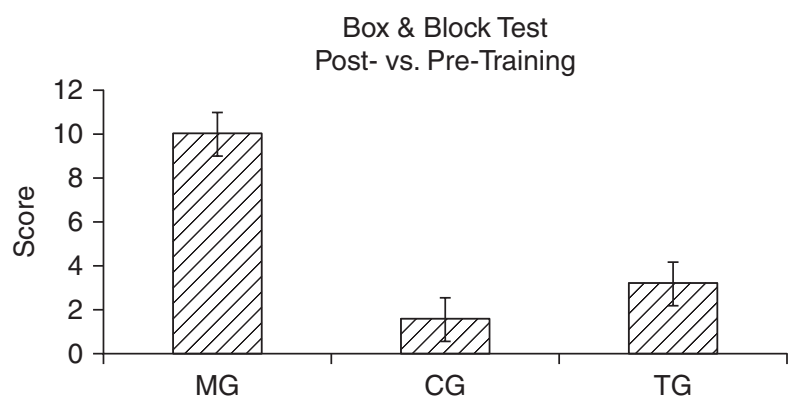

FIGURE 1. Results of the increase in transported cubes per minute in the Box and Block test following training (mean and standard deviation). sizes of 0.4 to 0.6 moderate, and effect sizes of 0.8 large. Effect sizes for MG patients for the Box and Block Test, Arm Paresis Score, finger tapping parameters, and hand tapping NIV were moderate. For the TG, small effect sizes were reached in the Hand tapping VMAX , Diadochokinesis Frequency, and VMAX. For the CG, all effect sizes were extremely small.

To assess subjective aspects of the music-supported training, patients from the MG and TG were asked to rate on a 5-point scale to what extent they benefited from the training (“optimal," "high," "medium," "some," or "none"). With regard to generalization of the training effect to every day functions in the MG, $69 \%$ of participants reported an optimal degree of transfer, while $18 \%$ rated their outcome in the high range and $13 \%$ in the medium range. In the TG patients, $53 \%$ of the participants reported an optimal degree of transfer, $40 \%$ rated their outcome in the high range, and $7 \%$ in the medium range.

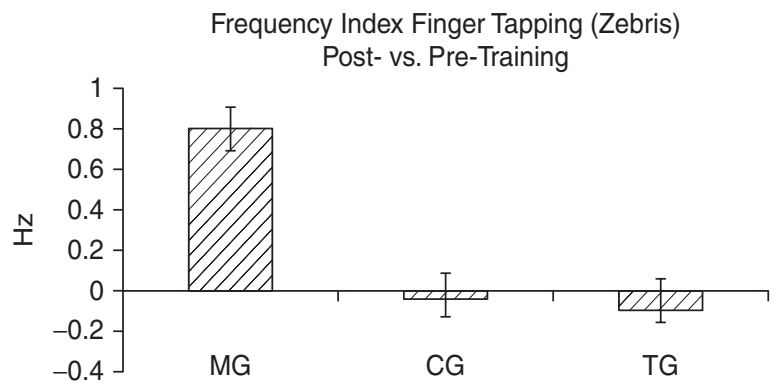

FIGURE 2. Results of the increase frequency of finger tapping following training (mean and standard deviation). 
TABLE 4. Effect Sizes of the Improvements Observed in MG, CG, and TG.

\begin{tabular}{|c|c|c|c|c|c|}
\hline Motor Test/Parameter & $\begin{array}{c}\text { Time-point } \\
\text { (Pre / Post) } \\
F(2,74)\end{array}$ & $\begin{array}{c}\text { Group } \mathrm{x} \\
\text { Time-point } \\
F(2,74)\end{array}$ & $\begin{array}{c}\text { MG } \\
\text { Cohen's } d\end{array}$ & $\begin{array}{c}\text { CG } \\
\text { Cohen's } d\end{array}$ & $\begin{array}{c}\text { TG } \\
\text { Cohen's } d\end{array}$ \\
\hline Box and Block Test (score) & $57.08^{\star *}$ & $19.94^{\star *}$ & 0.66 & 0.08 & 0.11 \\
\hline Nine Hole Pegboard Test (score) & $8.47^{\star}$ & $6.75^{\star}$ & 0.34 & 0.01 & 0.04 \\
\hline Action Research Arm Test(score) & $22.42^{\star *}$ & $10.49^{* *}$ & 0.39 & 0.02 & 0.14 \\
\hline Arm Paresis Score Wade (score) & $13.04^{\star}$ & $7.93^{*}$ & 0.59 & 0.04 & 0.11 \\
\hline Motor Test/Parameter & $\begin{array}{c}\text { Time-point } \\
\text { (Pre/ Post) } \\
F(2,66)\end{array}$ & $\begin{array}{c}\text { Group } \mathrm{x} \\
\text { Time-point } \\
F(2,66)\end{array}$ & $\begin{array}{c}\text { MG } \\
\text { Cohen's } d\end{array}$ & $\begin{array}{c}\text { CG } \\
\text { Cohen's } d\end{array}$ & $\begin{array}{c}\text { TG } \\
\text { Cohen's } d\end{array}$ \\
\hline Finger Tapping FREQ in $\mathrm{Hz}$ & $7.93^{*}$ & $15.56^{\star *}$ & 0.54 & 0.02 & 0.07 \\
\hline Finger Tapping VMAX in $\% s$ & 1.73 & $6.66^{*}$ & 0.54 & 0.12 & 0.04 \\
\hline Finger Tapping NIV & 1.04 & $7.23^{\star}$ & 0.55 & 0.15 & 0.02 \\
\hline Hand Tapping FREQ in $\mathrm{Hz}$ & $9.23^{*}$ & $7.55^{\star}$ & 0.45 & 0.04 & 0.14 \\
\hline Hand Tapping VMAX in $\% s$ & 0.11 & $5.19^{*}$ & 0.46 & 0.04 & 0.21 \\
\hline Hand Tapping NIV & 1.83 & $5.52^{\star}$ & 0.52 & 0.18 & 0.10 \\
\hline Diadochokinesis FREQ in $\mathrm{Hz}$ & $4.69^{*}$ & 2.22 & 0.37 & 0.02 & 0.24 \\
\hline Diadochokinesis VMAX in $\% s$ & 1.14 & 1.18 & 0.07 & 0.07 & 0.38 \\
\hline Diadochokinesis NIV & $4.92^{*}$ & $4.24^{\star}$ & 0.60 & 0.03 & 0.04 \\
\hline Aiming $5 \mathrm{~cm} \mathrm{V2} \mathrm{in} \mathrm{mm} / \mathrm{s}$ & $11.40^{*}$ & $9.85^{\star *}$ & 0.62 & 0.13 & 0.16 \\
\hline Aiming $0.8 \mathrm{~cm} \mathrm{~V} 2$ in $\mathrm{mm} / \mathrm{s}$ & $7.49^{*}$ & $4.67^{\star}$ & 0.41 & 0.04 & 0.18 \\
\hline
\end{tabular}

${ }^{* *} p<.001,{ }^{*} p<.05$.

\section{Discussion}

Previously published data have already shown positive effects of music-supported training (Altenmüller et al., 2009; Schneider et al., 2007). The present work extends these findings to a larger group of patients suffering from incomplete paresis of an upper extremity and shows that fifteen 30-minute sessions of music-supported training in addition to conventional treatment not only improve motor performance but also seem to be more efficient in terms of recovery of fine motor functions compared to a CIT of skilled movements according to Taub et al. (1993). We admit that due to the later recruitment of the CIT group, a fully randomized prospective study was not conducted. However, we are confident that any bias with respect to patient recruitment was avoided as carefully as possible.

It may seem odd that the control group, which received 28 units of conventional treatment on average, did not show improvement in any of the measures. However, this null effect corroborates previous observations (e.g., Woldag \& Hummelsheim, 2002). Thus, the current study is not unique in casting doubt on the efficiency of conventional therapies in the remediation of motor deficits after stroke.
One important issue that arose from our previous studies is to which degree the effects observed in the present study are due to specific aspects of the training; namely, practice with musical instruments or unspecific aspects such as practice of skilled finger movements. Our study offers the first evidence that music-supported training is more efficient than functional motor training with regards to finger dexterity and to the four clinical test batteries applied. Interestingly, gross motor movements requiring supination and pronation of the forearm for the diadochokinesis task seem not to benefit substantially more from music-supported training compared to functional motor training. This may be due to the fact that these movements were not included in either music making paradigm, whereas single finger movements as well as tapping movements of the wrist constituted parts of the instrumental training at the piano or the drum pads respectively. With respect to the overall efficacy of the music-supported training, it should be mentioned that most of the effect sizes range between 0.45 and 0.66 . Thus, our training seems to be comparably effective as the constrained induced movement therapy (CIMT) training, which is mostly considered the gold standard in rehabilitation of fine motor control. The CIMT approach involves massed practice with: (1) motor restriction of the unaffected 
upper extremity by a resting hand splint/sling for a period of 12 days, and (2) training of the affected extremity by a procedure termed "shaping" (i.e., execution of a battery of motor tasks with increasing level of difficulty) for approximately six hours per day for eight weekdays. Sterr and colleagues (2002) investigated the efficiency of CIMT and concluded that the effective factor in the CIMT appears to be the repeated use of the paretic arm for many hours per day for a period of several consecutive days, while the mechanical restriction of the healthy extremity seemed less important (see also Sterr \& Freivogel, 2003). With regard to the "dose" of repetitive movements, Sterr and Freivogel (2003)'s CIMT protocol called for 6, 3, or 1.5 hours per day of practice, which compares to "only" fifteen 30-minute sessions in the present study. Standard CIMT treatment protocols have yielded effect sizes of 0.46 (van Peppen, 2004) and between 0.34 and 0.45 (van der Lee, 2001) for dexterity of the paretic arm. Whereas the duration of the treatment session was considerably shorter in the current study, music-supported training nevertheless resulted in effect sizes of up to 0.6. This suggests that in addition to the intensity aspect, other factors might be instrumental for the effectiveness of music-supported training.With respect to the neurobiological mechanisms, audio-motor coupling may constitute a major element of the efficacy of music-supported training after stroke. To perform music, a high-speed mechanism is needed to control complex movement patterns under continuous auditory feedback. As a prerequisite, audio-motor integration at cortical and probably subcortical levels must be accomplished. This audio-motor coupling is established during the learning and training phases and could be compared to the oral-aural loop in language processing. A number of recent studies attest to the rapid effects of audio-motor coupling during music making in novice participants (Bangert \& Altenmüller, 2003; Lahav et al., 2007; Meyer, Elmer, Baumann \& Jäncke 2007).

In a previous study with a smaller group of patients undergoing music-supported training, an increase in neuronal connectivity between sensory-motor and auditory regions was demonstrated by means of EEG-coherence measures (Altenmüller et al., 2009). Therefore, it seems that similar mechanisms of establishing an audio-motor corepresentation-as observed in healthy adult novices who learned to play the piano-may play a role in the rehabilitation process (Bangert \& Altenmüller, 2003). This notion is corroborated by findings in a patient who underwent musicsupported training 20 months after suffering a stroke.
Along with clinical improvement, fMRI follow up provided evidence for the establishment of an auditorysensory motor network due to the training procedure (Rojo et al., 2010).

\section{Conclusions and Open Questions}

Undoubtedly, music-supported training is efficient and seems to be even more efficient than functional motor training. A randomized prospective study comprising all three groups is presently under the way and will clarify the differential effects of functional motor training and music-supported training. With respect to the underlying mechanisms, there still remain a number of open questions. First, which role motivational factors play must be clarified. From the patients informal descriptions of their experience with the music-supported training, it appears that this was highly enjoyable and a highlight of their rehabilitation process. Thus, motivational and emotional factors might have contributed to the success of the training program. Furthermore, according to a recent study by Särkämö and colleagues (2008), music listening activates a wide-spread bilateral network of brain regions related to attention, semantic processing, memory, motor functions, and emotional processing. Särkämö and colleagues showed that music exposure significantly enhances cognitive functioning in the domains of verbal memory and focused attention in a music group compared to a control group. The music group also experienced less depressed and confused mood than the control groups. These mechanisms may also hold true for the music-supported training we applied.

Another issue is related to the auditory feedback mechanisms. Up to now it has not been clear whether any auditory feedback (e.g., simple beep tones) would have a similar effect on fine motor rehabilitation or whether explicit musical parameters such as a sophisticated pitch and time structure are prerequisites for the success of the training. This will be addressed in a planned study comparing the effects of musical feedback compared to simple acoustic feedback. With respect to the latter, according to a study by Thaut, Kenyon, Hurt, McIntosh, and Hoemberg (2002), simple rhythmic cueing with a metronome significantly improves the spatio-temporal precision of reaching movements in stroke patients. Finally, the stability of improvements needs to be assessed in further studies, and the length and number of training sessions might be manipulated in future research. Additionally, the effect of training in chronic patients suffering from 
motor impairments following a stroke for more than a year will be assessed.

\section{Author Note}

This research was supported by grants from the DFG (AL 269/7-2). We thank the patients and the staff of NRZ Magdeburg, whose enthusiasm greatly facilitated the study. Michael Jöbges, M.D. was the consultant for movement analysis and Marc Bangert, Ph.D. programmed the MIDI-computer interface.

Correspondence concerning this article should be addressed to Dr. Eckart Altenmüller, Institute of Music Physiology and Musicians' Medicine, University of Music and Drama Hanover, Hohenzollernstraße 47, 30161 Hanover, Germany. E-MAIL altenmueller@hmthannover.de

\section{References}

Altenmüller, E., Schneider, S., Marco-Pallares, P. W., \& MÜnte, T. F. (2009). Neural reorganization underlies improvement in stroke induced motor dysfunction by music supported therapy. Annals of the New York Academy of Sciences, 169, 395-405.

Bangert, M., \& Altenmüller, E. (2003). Mapping perception to action in piano practice: A longitudinal DC-EEG-study. BMC Neuroscience, 4, 26-36.

Bangert, M., Peschel, T., Schlaug, G., Rotte, M., Drescher, D., Hinrichs, H., ET AL. (2006). Shared networks for auditory and motor processing in professional pianists: Evidence from fMRI conjunction. NeuroImage, 30, 917-926.

Воватн, B. (1948). The importance of reduction of muscle tone and the control of mass reflex action in the treatment of spasticity. Occupational Therapy and Rehabilitation, 27, 371-383

Buonomano, D. V., \& Merzenich, M. M. (1998) Cortical plasticity: From synapses to maps. Annual Revue of Neuroscience, 21, 149-186.

CoHen, J. (1988) Statistical power analysis for the behavioural sciences (2nd ed.). Hillsdale, NJ: Lawrence Earlbaum Associates.

Heller, A., Wade, D. T., Wood, V. A., Sunderland, A., Langton-Hewer, R., \& Ward, E. (1987). Arm function after stroke: Measurement and recovery over the first three months. Journal of Neurology, Neurosurgery, and Psychiatry, 50, 714-719.

Hermsdörfer, J., Marquardt, C., Wack, S., \& Mai, N. (1999). Comparative analysis of diadochokinetic movements. Journal of Electromyography and Kinesiology, 9, 283-295.

Kujala, T., Alho, K., \& NaAtanen, R. (2000). Cross-modal reorganization of human cortical functions. Trends in Neuroscience, 23,115-120.

Lahav, A., Saltzman, E., \& Schlaug, G. (2007). Action representation of sound: audiomotor recognition network while listening to newly acquired actions. Journal of Neuroscience, 27, 308-314.

Langhammer, B., \& Stanghelle, J.K. (2000). Bobath or motor relearning programme? A comparison of two different approaches of physiotherapy in stroke rehabilitation: A randomised controlled study. Clinical Rehabilitation, 14, 361-369.

Liepert, J., Bauder, H., Miltner, W. H. R., Taub, E., \& WeILler, C. (2000). Treatment-induced cortical reorganization after stroke in humans. Stroke, 31, 1210-1216.

LYLE, R. C. (1981). A performance test for assessment of upper limb function in physical rehabilitation treatment and research. International Journal of Rehabilitation Research, 4, 483-492.

Mahoney, F., \& Barthel, D. (1965). Functional evaluation: The Barthel index. Maryland State Medical Journal, 14, 61-65. Mathiowetz, V., Volland, G., Kashman, N., \& Weber, K. (1985). Adult norms for the Box and Block Test of Manual Dexterity. American Journal of Occupational Therapy, 39, 386-391.

Meyer, M., Elmer, S., Baumann, S., \& Jäncke, L. (2007). Short-term plasticity in the auditory system: Differential neural responses to perception and imagery of speech and music. Restoration, Neurology and Neurosciences, 25, 411-431.

Parker, V. M., Wade, D. T., \& Langton-Hewer, R. (1986). Loss of arm function after stroke: Measurement, frequency and recovery. International Journal of Rehabilitation Medicine, $8,69-73$.

Rojo, N., Amengual, J., Juncadella, M., Camara, E., Marco-Pallares, J., Rubio, F., Et Al. (2010). Musicsupported therapy induces plasticity in the sensorimotor cortex in chronic stroke: A single case study using multimodal imaging ( $\mathrm{fMRI-TMS).} \mathrm{Manuscript} \mathrm{submitted} \mathrm{for} \mathrm{publication.}$

Rossini, P. M., \& PAURI, F. (2000). Neuromagnetic integrated methods tracking human brain mechanism of sensorimotor areas "plastic" reorganization. Brain Research Review, 33, 131-154.

Särkämö, T., Tervaniemi, M., Laitinen, S., Forsblom, A., Soinila, S., Mikkonen, M., ET AL. (2008). Music listening enhances cognitive recovery and mood after middle cerebral artery stroke. Brain, 131, 866-876.

Schneider, S., Schönle, P. W., Altenmüller, E., \& MÜnTE, T. F. (2007). Using musical instruments to improve motor skill recovery following a stroke. Journal of Neurology, 254., 1339-1346. 
Sterr, A., Elbert, T., Berthold, I., Kolbel, S., Rockstroh, B., \& Taub, E. (2002). Longer versus shorter daily contraint-induced movement therapy of chronic hemiparesis: An exploratory study. Archives of Physical and Medical Rehabilitation, 830, 1374-1377.

Sterr, A., \& Freivogel, S. (2003). Motor-improvement following intensive training in low-functioning chronic hemiparesis. Neurology, 61, 842-844

Taub, E., Miller, N. E., Novack, T. A., Cook, E. W., Fleming, W. C., Nepomuceno, C. S., et al. (1993). Technique to improve chronic motor deficit after stroke. Archives of Physical and Medical Rehabilitation, 74, 347-374.

Thaut, M., Kenyon, G. P., Hurt, C. P., McIntosh, G. C., \& Hoemberg, V. (2002). Kinematic optimization of spatiotemporal patterns in pare training with stroke patients. Neuropsychologia, 40, 1073-1081.

VAN DER LEe, J. H. (2001) Constraint-induced therapy for stroke: More of the same or something completely different? Current Opinions in Neurology, 14, 741-744.

VAN Peppen R. P. S. (2004) The impact of physical therapy on functional outcomes after stroke: What's the evidence? Clinical Rehabiltation, 18, 833-862.

Wade, D. T., Langton-Hewer, R., Wood, V. A., Skilbeck, C. E., \& Ismail, H. M. (1983). The hemiplegic arm after stroke: Measurement and recovery. Journal of Neurology Neurosurgery Psychiatry, 46, 521-524.

Woldag, H., \& Hummelsheim, H. (2002). Evidence-based physiotherapeutic concepts for improving arm and hand function in stroke patients. Journal of Neurology, 249, 518-528. 